\title{
COVID-19-Pandemie - Rationalität statt Regelungschaos tut not
}

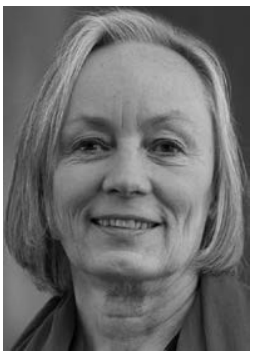

Ursel Heudorf
Bibliografie

Gesundheitswesen 2020; 82: 941-943

DOI $10.1055 / \mathrm{a}-1288-7064$

ISSN $\quad 0941-3790$

(c) 2020. Thieme. All rights reserved.

Georg Thieme Verlag KG, Rüdigerstraße 14,

70469 Stuttgart, Germany

Korrespondenzadresse

Prof. Dr. Ursel Heudorf

MRE-Netz Rhein-Main c/o Gesundheitsamt

Breite Gasse 28

60313 Frankfurt am Main

Deutschland

mre-rhein-main@stadt-frankfurt.de
Die COVID-19-Politik (nicht das Virus SARS-CoV-2 selbst) hat das Land im Griff. Mit den seit Anfang Oktober steigenden Infektionszahlen werden Regelungen über Regelungen erlassen von Sperrstunde, Versammlungseinschränkungen, verschärfter Maskenpflicht bis hin zum Beherbergungsverbot - verbunden mit der Drohung, dass, wenn dies nicht wirke, ein neuer Lockdown unerläßlich sei. Ohne die sich abzeichnende Wirkung der vorherigen Maßnahmen abzuwarten, beschließt die Politik rasch einen weiteren sog. „Lockdown light“. Die Gesundheitsämter schaffen die Nachverfolgung nicht mehr? Kein Problem: die Politik schickt Soldaten. Die Ordnungsämter sind mit der Kontrolle der vielen Verordnungen überfordert? Kein Problem: die Politik schickt die Bundespolizei.

Regelungschaos und Aktionismus in weiten Teilen der Politik. Gerichte mussten mehrfach die Reißleine ziehen. Aber: Nicht immer mehr Regelungen tun not, sondern Rationalität. Was sind die Ziele? Welche Lehren können (und müssen) aus den Erfahrungen früherer Pandemien gezogen werden?

Die Argumentation, bei SARS-CoV-2 handele es sich um ein neues Virus, über das man vieles noch nicht wisse, weshalb man nicht auf bisherigen Erfahrungen aufbauen könne, zieht nicht. Pandemie-Viren sind immer völlig neuartige Viren, die es zuvor nicht gab, die leicht übertragbar sind und die auf eine „immunologisch naive“ Weltbevölkerung treffen. Wäre das nicht so, könnten sie keine Pandemie auslösen. Somit handelt es sich bei SARS-CoV-2 um ein durchaus ernst zu nehmendes neuartiges Virus - aber nicht um ein neues Phänomen.

\section{Nationaler Pandemieplan}

Der Nationale Pandemieplan in Deutschland beschreibt in Übereinstimmung mit anderen Pandemieplänen (z. B. WHO) verschiedene Phasen und Ziele der Pandemiebekämpfung: Containment (Eindämmungsstrategie), Protection (Schutz vulnerabler Gruppen), Mitigation (Folgenminderung) und Recovery (Erholung) [1]. Ein Blick hinein lohnt sich: „Frühe Erkennung und Eindämmung/Beein- flussung der Ausbreitungsdynamik („detection \& containment“): Bei dem Auftreten eines neuen Mensch-zu-Mensch übertragbaren Influenzavirus fokussiert die Strategie auf die frühe Erkennung von einzelnen Infektionen und auf Maßnahmen, die die Verbreitung des Influenzavirus möglichst lange verzögern“ [1].

Die Erfahrung aus früheren Pandemien zeigt, dass das Containment ab einer gewissen Ausbreitung eines neuartigen leichtübertragbaren Virus nicht mehr funktionieren kann, insbesondere, wenn das Virus viele asymptomatische und leichte Infektionen verursacht und die Infizierten unerkannt zu Überträgern werden. Deswegen sind in den Pandemieplänen weitere Phasen der Pandemiebekämpfung vorgesehen: 2) Protection oder Schutz vulnerabler Gruppen: „Die Schutzmaßnahmen werden auf die Personengruppen konzentriert, die ein erhöhtes Risiko für schwere und tödliche Krankheitsverläufe haben, sowie Personen, die engen Kontakt zu diesen Personen haben, z. B. medizinisches Personal, und 3) Mitigation oder Folgenminderung: Wenn eine anhaltende Mensch-zu-Mensch-Übertragung in der Bevölkerung in Deutschland stattfindet, haben die eingesetzten Schutzmaßnahmen vor allem das Ziel, schwere Krankheitsverläufe zu verhindern und Krankheitsspitzen mit einer Überlastung der Versorgungsstrukturen zu vermeiden" [1].

Das ist keine Kapitulation vor dem Virus, sondern rationales Handeln und Einsicht in die Realität. Nur so kann Schaden von der Bevölkerung mit größtmöglichen Erfolgsaussichten abgewendet werden.

\section{Lehren aus früheren Pandemien}

Wesentliche Erfahrungen aus der Influenzapandemie 2009 wurden in einem Schwerpunktheft des Bundesgesundheitsblatts „Pandemien lessons learned“ niedergelegt [2-4].

„In den Monaten Mai bis Juli erfolgte die Diagnostik überwiegend aufgrund einer epidemiologischen Indikation und war eine Entscheidungsgrundlage für Isolierungsmaßnahmen. Eine fließende Anpassung der Strategie auf Basis der aktuell vorliegen- 
den Daten erfolgte ab Juli 2009. Die Isolierungsmaßnahmen wurden zunehmend nur noch auf Personen angewandt, die andere besonders gefährdete Personen betreuen, und schließlich bei steigender Anzahl autochthoner Fälle, wurde nur noch allen Erkrankten empfohlen, bis mindestens einen Tag nach Abklingen der Symptome zu Hause zu bleiben. ...Die Handlungsstrategie wurde nach Beobachtung des Anstiegs autochthoner Fälle in allen Bundesländern dahingehend geändert, die Auswirkungen der Pandemie bestmöglich zu bewältigen “ [2].

Als Mangel wurde beschrieben, dass die Meldedaten alleine für die zu treffenden Entscheidungen nicht ausreichen, und es wurden u. a. geeignete Krankenhaussurveillance- oder Sentinelsysteme sowie Erfassungssysteme für Mortalität, für schwere klinische Verläufe, für die Auslastung der Versorgungsstrukturen gefordert [2,3].

\section{9 fand eine „paternalistische“ Kommunikation statt mit dem}

\section{„Ziel, die Bürger anzuleiten, die vermeintlich richtige Entschei- dung zu treffen, und nicht, Bürger zu informieren, damit sie selbständig rationale Entscheidungen treffen können. ... Eine paternalistische Informationspolitik, die Menschen selektiv informiert und Unsicherheiten vorenthält, resultiert (1) in falscher Sicherheit oder gar Verunsicherung durch widersprüchliche Informationen und, (2) falls sich die Informationspolitik im Nachhinein als unangebracht herausstellt, in Ärger und Ver- schwörungstheorien “. Im Hinblick auf weitere Pandemien in der Zukunft wurde ein Umdenken gefordert einschließlich einer „Informationspolitik, die die Voraussetzungen für aufgeklärte und informierte Bürger schafft" [4].}

Und heute? Im Gegensatz zu den im bewährten Pandemieplan festgelegten Phasen bleibt die Politik der ersten Phase Containment verhaftet. Wider besseres Wissen und gegen alle Regeln der Pandemiebekämpfung müssen die Gesundheitsämter weiter mit großem Aufwand positiven (oder vermeintlich positiven) Fällen nachgehen und Einschränkungen der Grundrechte verfügen.

Dabei hat man aus der Pandemie 2009 gelernt: Die damals geforderten Surveillance-Systeme stehen heute u. a. mit den Systemen wie ARE, Grippeweb, SARI, aber auch dem DIVI-Intensivregister oder dem Integrierten Versorgungsnachweis IVENA und - in Hessen und Berlin - mit EUROMOMO zur Verfügung. Noch nie gab es so gute und umfassende Surveillance-Daten.

Die politischen Entscheidungsträger und die Medien nutzen aber weiterhin nahezu ausschließlich die Zahl der positiv auf SARS-CoV-2 Getesteten. In Reaktion darauf wird ihnen entgegengehalten, mit dieser „Mutter aller Zahlen“ Angst zu schüren und gegenüber der vermeintlich uneinsichtigen Bevölkerung ein Bedrohungsszenario zu beschwören. Mit der erneut angewandten paternalistischen Informationspolitik „verlieren“ sie die Bevölkerung. Angst schlägt irgendwann in Hilflosigkeit und Passivität um. Zuviel Bevormundung, zu viele Verbote führen zu Abwehr und Widerstand. Verunsicherung und Verschwörungstheorien sind die Folge.

\section{Und der Öffentliche Gesundheitsdienst?}

Zweifellos hat der Öffentliche Gesundheitsdienst (ÖGD) in den ersten Wochen und Monaten COVID-19 Pandemie in der Pandemiebekämpfung Hervorragendes geleistet. Dank seiner Arbeit konnten die initialen Ziele, die Pandemiekurve flach zu halten, um Zeit zu gewinnen, „hinter die Influenza-Zeit“ zu kommen und das Gesundheitswesen weiter auszubauen, erreicht werden. Dafür hat der ÖGD von der Politik viel Aufmerksamkeit und Wertschätzung erhalten. Gesundheitsminister Spahn legte bereits im April 2020 ein Papier „Stärkung des Öffentlichen Gesundheitsdienstes“ vor [5]. Die Politik beschloss im September 2020 einen „Pakt für den öffentlichen Gesundheitsdienst“ für eine bessere personelle und technische Ausstattung der Gesundheitsämter über die nächsten fünf Jahre [6].

Ein genaueres Hinsehen zeigt: Das Papier „Stärkung des öffentlichen Gesundheitsdienstes “ [5] fokussiert ausschließlich auf Meldewesen, Kontaktnachverfolgung, Isolierung und Quarantänisierung sowie die Digitalisierung des Meldewegs. Es sollen Kontaktverfolgungsteams von 5 Personen pro 20000 Einwohner gebildet werden, z. B. durch Abordnungen aus anderen Bereichen der Verwaltung oder der Bundeswehr, mit „Containment-Scouts“ des Robert Koch-Instituts oder mit „Medis4ÖGD“. Kein Wort zu anderen wichtigen präventiven Aufgaben des ÖGD. Statt den ÖGD zu stärken, birgt diese Fokussierung die Gefahr der Abwendung von einem auch in seinen anderen Aufgabenfeldern modernen und zukunftsfähigen ÖGD, wie er im Leitbild ÖGD beschrieben ist [7].

In der langen und wechselvollen Geschichte des ÖGD und nach verschiedenen großen Reformdebatten wurde dieses Leitbild im Jahr 2018 verabschiedet [7, 8]. In diesem Leitbild des ÖGD finden sich neben den bekannten Kernaufgaben wie Infektionsschutz, Impfung, Hygieneberatung und Kontrolle, Trinkwasserhygiene usw. viele steuernde, partizipative und gesundheitsfördernden Aufgaben: niedrigschwellige Angebote und aufsuchende Gesundheitshilfen wie bspw. „Frühe Hilfen“, sozialpsychiatrische Beratung usw. Diese und die Koordination von Gesundheitsprojekten in der Kommune, Kommunikation, Gesundheitsberichterstattung und Politikberatung sind nicht nur in dem Leitbild, sondern oft auch als gesetzliche Aufgaben in den Gesundheitsdienstgesetzen der Länder verankert. Der „Pakt für den öffentlichen Gesundheitsdienst“ bezieht sich zwar durchaus auf das Leitbild - in der öffentlichen Kommunikation wird jedoch weiterhin der Fokus auf die Pandemiebekämpfung gelegt.

Die derzeitige, auf COVID-19 eingeengte „Wertschätzung“ des ÖGD durch die politischen Entscheidungsträger geht einher mit der realistischen Gefahr einer Degradierung des ÖGD auf eine Rolle als Virusjäger und Quarantänisierer, die dann konsequenterweise auch gleich weitgehend von angelernten Kräften übernommen werden kann. Die demgegenüber deutlich breitere Fachkompetenz des ÖGD, seine Erfahrung in der Pandemiebekämpfung und auf vielen weiteren Bereichen wird von der Politik nicht gehört.

Anfang September 2020 haben erfahrene Fachärzte für öffentliches Gesundheitswesen unter Hinweis auf den Pandemieplan und auf die Kriterien guten Verwaltungshandelns mit Prüfung der Legitimität, der Geeignetheit, Erforderlichkeit und Angemessenheit von einschränkenden Maßnahmen dringend eine breite öffentliche Diskussion zu den Zielen und Mitteln der Pandemie-Bekämpfung 
angemahnt - unter Berücksichtigung ethischer (primum non nocere) und rechtlicher Fragen (Primat des mildesten Mittels) $[9,10]$. Ärzte und Fachleute lobten den Beitrag und bewunderten den Mut der Autoren - offenbar braucht es heute Mut, Zahlen in ihrem Gesamtzusammenhang darzulegen und Fragen zu stellen? Auf der anderen Seite gab es die Phalanx derer, die - in seit Monaten „bewährter" Weise - den Autoren Verharmlosung vorwarfen, sie in eine bestimmte politische Ecke stellten und so eine Auseinandersetzung mit den Argumenten verhinderten. Die politischen Entscheidungsträger ignorierten diese Anregungen vollends.

Der ÖGD steht mit diesen Vorschlägen nicht alleine; auch viele andere Fachgesellschaften und Expertenkreise haben seit Monaten einen anderen Umgang mit der Pandemie angemahnt [u.a. [11-13].

Dennoch: Der ÖGD ist im Rahmen der ihm gesetzlich übertragenen Fachaufgaben der Gesundheit der Bevölkerung verpflichtet, der Gesundheit in einem ganzheitlichen Sinn. Der ÖGD muss nicht nur auf den Pandemieplan, sondern auch auf andere Gesundheitsrisiken hinweisen, wie bspw. die jährlich ca. 30000 Todesfälle durch ambulant erworbene Pneumonien, die Problematik der Hitzesterblichkeit oder von Depression und Suiziden in der Gesellschaft etc., für die er gemeinsam mit anderen Präventionskonzepte entwickelt hat, für deren Umsetzung er aber angesichts der derzeit geforderten Fokussierung auf SARS-CoV-2 keine Kapazität mehr hat. Die im November 2020 erneut in Kraft getretenen Einschränkungen auch der verantwortlich durchgeführten Sport- und Bewegungsangebote für Kinder und Erwachsene (Vereinssport, Fitness-Studios, Reha-Sport, Schwimmen, Bewegung in Freizeitparks) und aller kulturellen Angebote sind im Sinne der Prävention und Gesunderhaltung der Bevölkerung (physisch und psychisch) kontraproduktiv und konterkarieren etablierte Präventionsaufgaben des ÖGD.

„Der ÖGD ist gelebte Prävention. ... Diese beginnt bei der seuchenhygienischen Prävention, ist aber notwendiger Weise auch auf eine breite, bevölkerungsbezogene Prävention auszudehnen, die der ÖGD anwaltlich in unserer Gesellschaft als Zukunftsaufgabe für ein gesünderes Leben und Überleben übernehmen soll“ [14]. Nur so, als Anwalt der Bevölkerung und gehörter (!) Politikberater kann der ÖGD im Sinne der Gesundheit der Bevölkerung wirken.

Interessenkonflikt

Die Autorinnen/Autoren geben an, dass kein Interessenkonflikt besteht.
Literatur

[1] Nationaler Pandemieplan Teil I Struktiuren und Massnahmen RKI 2017 https://www.gmkonline.de/documents/ pandemieplan_teili_1510042222_1585228735.pdf

[2] Marcic A, Dreesmann J, Liebl B et al. H1N1-Pandemie. Maßnahmen und Erfahrungen auf Landesebene. Bundesgesundheitsblatt Gesundheitsforschung Gesundheitsschutz 2010; 53: 1257-1266

[3] Schaade L, Reuß A, Haas W et al. Pandemieplanung. Was haben wir aus der Pandemie (H1N1) 2009 gelernt? Bundesgesundheitsblatt Gesundheitsforschung Gesundheitsschutz 2010; 53: 1277-1282

[4] Feufel MA, Antes G, Gigerenzer G. Vom sicheren Umgang mit Unsicherheit: was wir von der pandemischen Influenza (H1N1) lernen können. Bundesgesundheitsblatt Gesundheitsforschung Gesundheitsschutz 2010; 53: 1283-1289

[5] BMG. Stärkung des Öffentlichen Gesundheitsdienstes. 21.04.2020 https://oegd.gmp-podcast.de/podcast/oegd029/

[6] BMG Pakt für den öffentlichen Gesundheitsdienst 05.09.2020 https:// www.bundesgesundheitsministerium.de/fileadmin/Dateien/3_ Downloads/O/OEGD/Pakt_fuer_den_OEGD.pdf

[7] Gesundheitsministerkonferenz: Leitbild für einen modernen öffentlichen Gesundheitsdienst (ÖGD) - „Der ÖGD: Public health vor Ort“ 2018. http://www.bvoegd.de/leitbild/

[8] Kuhn J, Wildner M. Corona-Krise und öffentlicher Gesundheitsdienst. GGW 2020; 20 Heft 4 Oktober, 15-22

[9] Heudorf U, Gottschalk R. Die Covid-19-Pandemie in Frankfurt am Main: Was sagen die Daten? Hessisches Ärzteblatt 2020; 81: 548-550

[10] Gottschalk R, Heudorf U. Die Covid-19-Pandemie - bisherige Erkenntnisse und Empfehlungen für das weitere Vorgehen. Hessisches Ärzteblatt 2020; 81: 551-552

[11] Stellungnahme der DGKH, DGPI, DAKJ, GHUP und bvkj. Kinder und Jugendliche in der COVID-19-Pandemie: Schulen und Kitas sollen wieder geöffnet werden. Der Schutz von Lehrern, Erziehern, Betreuern und Eltern und die allgemeinen Hygieneregeln stehen dem nicht entgegen 19.05.2020 https://www.krankenhaushygiene.de/ccUpload/ upload/files/2020_05_19_Stellungnahme_DGKH_Covid-19_Kinder. pdf

[12] Schrappe M, Francois-Kettner H, Gruhl M et al. Thesenpapiere 1.0-5.0 zu Corona. http://www.matthias.schrappe.com/akt24.htm

[13] Bundesvereinigung Kassenärztliche, Streek H, Schmidt-Chanasit J et al Gemeinsame Position von Wissenschaft und Ärzteschaft. Evidenz- und Erfahrungsgewinn im weiteren Management der COVID-19-Pandemie berücksichtigen. https://www.kbv.de/media/sp/2020-10-28_KBVPositionspapier_COVID-19.pdf

[14] Gruhl M.Quo vadis ÖGD? https://observer-gesundheit.de/quo-vadisoegd/ 